\title{
Experimental targets for photon couplings of the QCD axion
}

\author{
Prateek Agrawal, ${ }^{a}$ JiJi Fan, ${ }^{b}$ Matthew Reece ${ }^{a}$ and Lian-Tao Wang ${ }^{c}$ \\ ${ }^{a}$ Department of Physics, Harvard University, \\ 17 Oxford Street, Cambridge, U.S.A. \\ ${ }^{b}$ Department of Physics, Brown University, \\ 184 Hope Street, Providence, U.S.A. \\ ${ }^{c}$ Department of Physics, University of Chicago, \\ 5720 S Ellis Ave, Chicago, U.S.A. \\ E-mail: prateekagrawal@fas.harvard.edu, jiji_fan@brown.edu, \\ mreece@physics.harvard.edu, liantaow@uchicago.edu
}

ABSTRACT: The QCD axion's coupling to photons is often assumed to lie in a narrow band as a function of the axion mass. We demonstrate that several simple mechanisms, in addition to the photophilic clockwork axion already in the literature, can significantly extend the allowed range of couplings. Some mechanisms we present generalize the KNP alignment scenario, widely studied as a model of inflation, to the phenomenology of a QCD axion. In particular we present KSVZ-like realizations of two-axion KNP alignment and of the clockwork mechanism. Such a "confinement tower" realization of clockwork may prove useful in a variety of model-building contexts. We also show that kinetic mixing of the QCD axion with a lighter axion-like particle can dramatically alter the QCD axion's coupling to photons, differing from the other models we present by allowing non-quantized couplings. The simple models that we present fully cover the range of axion-photon couplings that could be probed by experiments. They motivate growing axion detection efforts over a wide space of masses and couplings.

KEYwords: Beyond Standard Model, Nonperturbative Effects

ArXiv ePrint: 1709.06085 


\section{Contents}

1 Introduction 1

2 Scenario I: alignment mechanism 4

2.1 A UV completion based on one confining hidden gauge group 5

$\begin{array}{lll}2.2 & \text { Landau pole constraint } & 8\end{array}$

3 Scenario II: confinement tower $\quad 8$

$\begin{array}{lll}3.1 & \text { Axion quality } & 10\end{array}$

4 Scenario III: kinetic mixing of multiple axions 11

$\begin{array}{lll}4.1 & \text { Realizing large mixing } & 12\end{array}$

5 Results and conclusions $\quad 13$

$\begin{array}{ll}\text { A Mass mixing and non-quantized couplings } & 15\end{array}$

$\begin{array}{lll}\text { B Two-loop RG equations for the model in section } 2 & 17\end{array}$

$\begin{array}{ll}\text { C Vector-like leptons with large PQ charge } & 18\end{array}$

$\begin{array}{ll}\text { C.1 A chain of vector-like fermions } & 19\end{array}$

$\begin{array}{lll}\text { C. } 2 \text { Clockwork } & 19\end{array}$

\section{Introduction}

The QCD axion is the most appealing simple solution to the strong CP problem [1-8] as well as a classic dark matter benchmark [9-11]. Given its very weak coupling to the standard model, searches to discover it have proved to be challenging. Yet experimental efforts have been growing very rapidly recently [12-18] with several of them aiming at detecting axion-photon couplings. It is thus important to chart the motivated parameter space for this coupling.

An axion is a periodic field, $a \cong a+2 \pi F_{a}$. This constrains its couplings to gauge fields, as $\theta$ has period $2 \pi$ in a coupling $\frac{\theta}{32 \pi^{2}} e^{2} F_{\mu \nu} \widetilde{F}^{\mu \nu}$, where the dual gauge field $\widetilde{F}_{\mu \nu}=\frac{1}{2} \epsilon_{\mu \nu \rho \sigma} F^{\rho \sigma}$. (Recall that even for a $\mathrm{U}(1)$ gauge theory, the $\theta$ term is physical, as manifested in the Witten effect [19].) Compatibility of the axion period and the $\theta$ angle period requires that when we have a coupling of an axion to gauge fields (abelian or nonabelian) of the form

$$
k \frac{\alpha}{8 \pi} \frac{a}{F_{a}} F_{\mu \nu} \widetilde{F}^{\mu \nu},
$$


with the gauge field canonically normalized, $\alpha=e^{2} /(4 \pi)$, and $e$ the coupling to a minimumcharge particle, the prefactor $k$ must be an integer. ${ }^{1}$

The QCD axion's mass is determined by nonperturbative dynamics resulting from its coupling to gluons,

$$
N \frac{\alpha_{s}}{8 \pi} \frac{a}{F_{a}} G_{\mu \nu}^{a} \widetilde{G}^{a \mu \nu}=\frac{\alpha_{s}}{8 \pi} \frac{a}{f_{a}} G_{\mu \nu}^{a} \widetilde{G}^{a \mu \nu} .
$$

Here $N$ is an integer and we have defined the effective decay constant

$$
f_{a} \equiv F_{a} / N
$$

In this paper our focus will be on the axion-photon coupling, $-\frac{g_{a \gamma \gamma}}{4} a F \widetilde{F}$. This is a sum of two contributions: the IR one from mixing between axion and QCD mesons [23-26], with

$$
g_{a \gamma \gamma}^{\mathrm{IR}}=-1.92(4) \frac{\alpha_{\mathrm{em}}}{2 \pi f_{a}},
$$

where $\alpha_{\mathrm{em}}$ is the electromagnetic coupling strength and $f_{a}$ is the effective decay constant introduced above. The number (4) indicates the NLO correction [27]. The UV contribution to the axion-photon coupling is model-dependent. It usually takes the form

$$
g_{a \gamma \gamma}^{\mathrm{UV}}=r \frac{\alpha_{\mathrm{em}}}{2 \pi f_{a}}, \quad \text { with } \quad r=\frac{E}{N},
$$

where $E$ and $N$ are the (discrete) electromagnetic and QCD anomaly coefficients of the PQ symmetry respectively. The IR contribution indicates the smallest size of the axionphoton coupling, provided that there is no accidental cancelation between the UV and IR contributions. In models where $E / N=2,{ }^{2}$ the axion-photon coupling is reduced by a factor of $\sim 20$ [28]. More extreme tuning is possible by considering multiple representations or through a kinetic mixing contribution. Notice that mixing of multiple axions can appear to evade the quantization rule (1.1), because the kinetic and mass terms may not be diagonal in a basis where the axion shift symmetries are diagonal. For clarity and pedagogical completeness, we elaborate on the origin of the non-quantized coupling (1.4) in appendix A.

The question is then: what is the upper bound of the QCD axion-photon coupling theoretically? Traditionally it is assumed that UV and IR contributions are of the same order and $g_{a \gamma \gamma} \sim \mathcal{O}(1) \alpha_{\mathrm{em}} /\left(2 \pi f_{a}\right)$. A variety of specific models realizing different $\mathcal{O}(1)$ coefficients have been used to define a standard band that is often plotted [29]. More thorough recent analyses demonstrate that in the standard KSVZ [5, 6] and DFSZ [7, 8] frameworks, it is true that $g_{a \gamma \gamma} \sim \mathcal{O}(1) \alpha_{\mathrm{em}} /\left(2 \pi f_{a}\right)$ for most representations of heavy matter charged under the PQ symmetry and the standard model gauge groups [30,31]. Yet special representations of KSVZ fermions and their combinations or multiple Higgses (9 Higgses)

\footnotetext{
${ }^{1}$ Because we normalize Standard Model charges so that the smallest is $1 / 3$ rather than 1 , the quantization of the coefficient is in units of $1 / 9$ rather than 1 . The periodicity of the theta angle can be altered by integer factors when the gauge group is quotiented by a discrete subgroup [20,21], and even in the Standard Model this leads to some ambiguity regarding the proper periodicity of the QED $\theta$ angle [22].

${ }^{2}$ This could be realized in specific models, e.g., a KSVZ model with one set of vector-like heavy quarks, which is a color fundamental and electroweak singlet, and one set of vector-like heavy leptons which only carry hypercharge $1(-1)$.
} 
with particular PQ charges in the DFSZ model could give rise to larger couplings [30, 31]. In this case, requiring that the Landau poles of the SM gauge couplings are above the Planck scale in the presence of the new matter charged under the SM gauge group, the QCD axion's coupling to photons could be maximally enhanced to 170/3 (KSVZ model) and 524/3 (DFSZ model). Recently it has also been proposed that axion coupling to photons could be enhanced exponentially in a clockwork axion scenario [32] (based on the clockwork idea of $[33,34]$, which had precursors in $[35,36])$. This clockwork photophilic axion relies on a particular structure of multiple scalars. It serves as a very interesting proof of concept that axion-photon couplings could be enhanced significantly.

In this article, we will showcase several different mechanisms that can achieve a large axion-photon coupling. Our goal is not just to prove that large couplings are possible, since the clockwork photophilic axion model already demonstrates that; rather, we aim to identify qualitatively different UV completions and explain the quantitative degree to which they can enhance the axion-photon coupling. Our main point will be that very simple extensions of familiar models of the QCD axion can lead to a substantial enhancement of $g_{a \gamma \gamma}$. The mechanisms include Kim-Nilles-Peloso (KNP) alignment [37] of two or more axions and kinetic mixing of multiple axions. We realize the KNP alignment mechanism with hidden confining gauge groups [33]. Models which iterate KNP alignment with a tower of confining gauge groups provide a useful realization of the clockwork mechanism. Among these mechanisms, the ones based on large PQ charge or alignment predict quantized couplings while kinetic mixing could give rise to non-quantized couplings. Together they motivate a much broader experimental parameter space for the QCD axion.

As a byproduct, we note that the KSVZ-like constructions of KNP alignment and clockwork models that we construct may be more generally useful for phenomenology. For the most part, KNP alignment has been discussed in the context of axions arising from extra-dimensional gauge fields, while clockwork was based on theories of many scalars with highly constrained quartic interactions. An alternative is a KSVZ-like approach to KNP alignment. We present a simple nonsupersymmetric realization of this idea. A similar supersymmetric construction appeared in [33], while a prototype of our nonsupersymmetric approach appeared recently in [38]. The version we present here differs in relying entirely on choices of gauge representations rather than a large number of fermion fields to obtain an enhancement. A rather different realization of clockwork based on a sequence of confining gauge groups also appeared recently in [39]; we will comment below on the similarities and differences to our approach.

In several mechanisms we present, new matter with standard model hypercharge will accelerate the running of the $\mathrm{U}(1)_{Y}$ gauge coupling. While the requiring the Landau pole to be above the Planck scale is not necessary, we will follow refs. $[30,31]$ to adopt it as a theoretical constraint. We also restrict that all the fields in the model to be have no higher than two-index representations of any non-Abelian gauge group. In part, this is because all of the physics we are interested in can be illustrated in simple models with only adjoint and fundamental representations. A further motivation is that models with light matter only in low-dimensional representations may be more UV completable. In D-brane models, one finds only two-index representations because a string has only two endpoints to attach 
to branes. In the heterotic string the story is more complicated, but similar statements are true at low Kac-Moody levels (see e.g. section 17.1 of [40]). In short, we expect that by avoiding large charge assignments we obtain easier compatibility with quantum gravity.

We do not consider in detail using large hypercharges (or equivalently a large number of fields with hypercharge) to boost the axion-photon coupling. In addition to being exotic without any dynamical reason, large hypercharges are also subject to the Landau pole constraint. Requiring the Landau pole of $\mathrm{U}(1)_{Y}$ to be above the Planck scale $\sim 10^{18} \mathrm{GeV}$ limits the hypercharge of the heavy matter to be $\lesssim 6$, which leads to an enhancement $\lesssim 100$. $^{3}$ A closely related possible method that may enhance the axion-photon coupling is to use large PQ charges. We will discuss it in appendix $\mathrm{C}$ and demonstrate that due to constraint on the heavy fermions' mass, the enhancement is also limited to be below 32 .

The same mechanisms that can be used to enlarge the QCD axion's coupling to photons could be used to enlarge the couplings to dark photons, which can help to make a wider range of QCD axion decay constants phenomenologically viable by altering the early universe cosmology [38]. More generally, the idea of alignment (through charges as in KNP or through kinetic mixing) has played a major role in recent models of inflation, but a relatively limited role in other particle physics phenomenology (though see [41, 42]). By illustrating simple renormalizable UV completions of alignment models, based on the same ideas as the original KSVZ axion model, we hope to spread these useful model-building tools to a wider phenomenological community.

\section{Scenario I: alignment mechanism}

The KNP alignment mechanism has been proposed and studied extensively in the natural inflation context $[36,37,43-47]$. In this section, we describe a KSVZ completion of the KNP alignment.

Let us first briefly review KNP alignment. In this scenario, we need at least two axion fields $a(x)$ and $b(x)$, both coupling to the gluons of a hidden gauge group $\mathrm{SU}(M)_{h}$ and QCD gluons. The basic mechanism could be described by the Lagrangian

$$
\begin{aligned}
\mathcal{L}= & -\frac{1}{4}\left(H_{\mu \nu} H^{\mu \nu}+G_{\mu \nu} G^{\mu \nu}+F_{\mu \nu} F^{\mu \nu}\right) \\
& +\frac{\alpha_{h}}{8 \pi F_{0}}\left(a+M^{\alpha} b\right) H_{\mu \nu} \widetilde{H}^{\mu \nu}+\frac{\alpha_{s}}{8 \pi F_{0}} b G_{\mu \nu} \widetilde{G}^{\mu \nu}+\frac{\alpha_{\mathrm{em}}}{8 \pi F_{0}} M^{\beta} a F_{\mu \nu} \widetilde{F}^{\mu \nu},
\end{aligned}
$$

where $H$ is the field strength of $\mathrm{SU}(M)_{h}$. The powers of $M$ in the anomaly coefficients, $\alpha, \beta \geq 1$, are some non-negative integer powers depending on the particle content of the model. Note that this Lagrangian is just illustrative. We have assumed that $a$ and $b$ have the same period $F_{0}$ for simplicity. We highlight the $M$ dependence and ignore $\mathcal{O}(1)$ numbers that could arise in a full model.

\footnotetext{
${ }^{3}$ The estimated number is obtained assuming a KSVZ model with one pair of vector-like quarks (without charge) and one pair of vector-like leptons which is only charged under $\mathrm{U}(1)_{Y}$. This upper bound holds as long as the fermion mass is below $10^{17} \mathrm{GeV}$.
} 


\begin{tabular}{|c|ccccc|}
\hline & $\mathrm{SU}(M)_{h}$ & $\mathrm{SU}(3)_{C}$ & $\mathrm{U}(1)_{Y}$ & $\mathrm{U}(1)_{P Q ; 1}$ & $\mathrm{U}(1)_{P Q ; 2}$ \\
\hline$\phi_{1}$ & 1 & 1 & 0 & -1 & 0 \\
$Q_{1 a}\left(\widetilde{Q}_{1 a}\right)$ & $\mathrm{Adj}$ & 1 & $1(-1)$ & $1(0)$ & $0(0)$ \\
$Q_{1 b}\left(\widetilde{Q}_{1 b}\right)$ & 1 & $3(\overline{3})$ & 0 & $1(0)$ & $0(0)$ \\
$Q_{1 c}\left(\widetilde{Q}_{1 c}\right)$ & $M(\bar{M})$ & 1 & 0 & $1(0)$ & $0(0)$ \\
\hline$\phi_{2}$ & 1 & 1 & 0 & 0 & -1 \\
$Q_{2 a}\left(\widetilde{Q}_{2 a}\right)$ & $\mathrm{Adj}$ & 1 & 0 & 0 & $1(0)$ \\
$Q_{2 b}\left(\widetilde{Q}_{2 b}\right)$ & 1 & $3(\overline{3})$ & 0 & 0 & $1(0)$ \\
\hline
\end{tabular}

Table 1. Particle content of a bi-axion alignment model.

The heavy hidden gauge group confines at a scale $\Lambda_{H} \gg \Lambda_{\mathrm{QCD}}$ and leads to a heavy axion, which is a linear combination of $a$ and $b$ (mostly $b$ ). Effectively we can set

$$
a+M^{\alpha} b=0 \Rightarrow b=-M^{-\alpha} a
$$

to integrate out the heavy axion. In the low energy effective theory, we find the couplings of the light axion $a$ to be,

$$
-\frac{\alpha_{s}}{8 \pi F_{0}} M^{-\alpha} a G \widetilde{G}+\frac{\alpha_{\mathrm{em}}}{8 \pi F_{0}} M^{\beta} a F \widetilde{F} .
$$

The first term suggests that the effective decay constant in this case is $f_{a}=M^{\alpha} F_{0}$, which could be significantly larger than the period $F_{0}$ in the UV theory. ${ }^{4}$ Then the QCD axion coupling to the photons is then enhanced by $M^{\alpha+\beta}$ :

$$
r \equiv g_{a \gamma \gamma}^{\mathrm{UV}}\left(\frac{\alpha_{\mathrm{em}}}{8 \pi f_{a}}\right)^{-1}=M^{\alpha+\beta} .
$$

Below we will show a simple KSVZ type model with $\alpha=1$ and $\beta=2$ such that the enhancement scales as $M^{3}$.

\subsection{A UV completion based on one confining hidden gauge group}

The particle content is shown in table 1 . The model is a variant of the KSVZ model. $\phi_{1}, \phi_{2}$

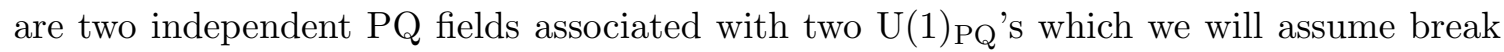
at the same scale $F_{0}$ for simplicity. Below $F_{0}$, there are two axion fields $a_{1}$ and $a_{2}$, which are the angular degrees of freedom of $\phi_{1}$ and $\phi_{2}$ respectively. We take the PQ charges of both $\phi_{1}$ and $\phi_{2}$ to be -1 . In addition, we have several sets of vector-like fermions. $Q$ and $\widetilde{Q}$ form a vector-like pair. All the fermions with a subscript 1, $Q_{1}$ 's, couple to $\phi_{1}$ while $Q_{2}$ 's couple to $\phi_{2}$, as implied by the PQ charge assignments: $y_{1} \phi_{1} Q_{1} \widetilde{Q}_{1}+y_{2} \phi_{2} Q_{2} \widetilde{Q}_{2}$. We also assume all the heavy fermions are weak singlets. The key feature of this model is that $\phi_{1}$ and $\phi_{2}$ couple to fermions with the same representations of $\mathrm{SU}(M)_{h}$ and $\mathrm{SU}(3)_{c}$ to

\footnotetext{
${ }^{4}$ In fact, because the light effective axion winds around the $(a, b)$ space, its period is also $f_{a}$ in the IR theory. We will treat these subtleties more carefully in the detailed example below.
} 
guarantee the alignment of the heavy axion and QCD axion, except for one set of fermion ( $Q_{1 c}$ in the specific example), which only couples to one of the PQ fields.

For convenience, because we will make use of it extensively below, we quote here the axion-gauge field coupling generated by integrating out massive fermions. A mass term $m(\phi) Q \widetilde{Q}$, with $m$ a general function of PQ-charged scalars and $Q, \widetilde{Q}$ in the $\mathbf{R}, \overline{\mathbf{R}}$ representations of the gauge group, produces a coupling

$$
\Delta \mathcal{L}=2 \mu(\mathbf{R}) \frac{g^{2}}{32 \pi^{2}} \arg (m) F_{\mu \nu}^{a} \widetilde{F}^{a \mu \nu},
$$

where $\mu(\mathbf{R})$ is the Dynkin index of the representation. For a U(1) gauge theory, $\mu(\mathbf{R})$ is simply $q^{2}$ with $q$ the quantized charge. Applying this general formula to our model, we have the axion couplings as

$$
\begin{aligned}
& \frac{\alpha_{h}}{8 \pi F_{0}}\left[2 \mu_{h}\left(Q_{1 a}\right)\left(a_{1}+a_{2}\right)+2 \mu_{h}\left(Q_{1 c}\right) a_{1}\right] H \widetilde{H}+\frac{\alpha_{s}}{8 \pi F_{0}}\left[2 \mu_{c}\left(Q_{1 b}\right)\left(a_{1}+a_{2}\right)\right] G \widetilde{G} \\
& \quad+\frac{\alpha_{\mathrm{em}}}{4 \pi F_{0}} D_{h}\left(Q_{1 a}\right) a_{1} F \widetilde{F} \\
& =\frac{\alpha_{h}}{8 \pi F_{0}}\left[2 M\left(a_{1}+a_{2}\right)+a_{1}\right] H \widetilde{H}+\frac{\alpha_{s}}{8 \pi F_{0}}\left(a_{1}+a_{2}\right) G \widetilde{G}+\frac{\alpha_{\mathrm{em}}}{4 \pi F_{0}}\left(M^{2}-1\right) a_{1} F \widetilde{F},
\end{aligned}
$$

where in the first two lines, $\mu$ 's $(D)$ are the Dynkin indices (dimension) of the corresponding $Q$ 's in the brackets.

To map this to the earlier more schematic discussion, note that $a_{1}+a_{2}$ plays the role of $b$ and $a_{1}$ plays the role of $a$ above. After integrating out the heavy combination $(2 M+1) a_{1}+2 M a_{2}$, the QCD axion $a$ is the light linear combination

$$
\begin{aligned}
a & =\frac{F_{0}}{F_{a}}\left[2 M a_{1}-(2 M+1) a_{2}\right], \quad \text { where } \\
F_{a} & =\sqrt{8 M^{2}+4 M+1} F_{0}
\end{aligned}
$$

sets the period of the light field, $a \cong a+2 \pi F_{a}$. In the large $M$ limit, the QCD axion is approximately $a_{1}-a_{2}$. Its period $F_{a}$ is larger than $F_{0}$ because the light field winds multiple times around the two-axion space; see e.g. figure 1 of ref. [36] for an illustration. The coupling to QCD determines the effective decay constant $f_{a}$ of the light field; in this case we find $f_{a}=F_{a}$, i.e. the number $N$ in equation (1.2) is 1. The QCD axion coupling to photons is enhanced by

$$
r=4 M\left(M^{2}-1\right)
$$

which could be of order 100-1000 for moderately large $M(3<M<10)$.

A few comments on model building are in order:

- In general, from the first line of eq. (2.6),

$$
r=\frac{D_{h}\left(Q_{1 a}\right) \mu_{h}\left(Q_{1 a}\right)}{\mu_{c}\left(Q_{1 b}\right) \mu_{h}\left(Q_{1 c}\right)} .
$$

To maximize $r, Q_{1 b}$ and $Q_{1 c}$ should be in the fundamental representations of $\mathrm{SU}(3)_{c}$ and $\mathrm{SU}(M)_{h}$ respectively, with the smallest possible Dynkin index $\mu=\frac{1}{2}$. $D$ and $\mu$ of 
the symmetric $k$-index representation of $\mathrm{SU}(M)$ are $\left(\begin{array}{c}M+k-1 \\ k\end{array}\right)$ and $\frac{1}{2}\left(\begin{array}{c}M+k \\ k-1\end{array}\right)$ respectively with the positive integer $k \geq 1$. Thus

$$
r=2\left(\begin{array}{c}
M+k-1 \\
k
\end{array}\right)\left(\begin{array}{c}
M+k \\
k-1
\end{array}\right), \quad k=1,2,3, \cdots
$$

If $Q_{1 a}$ and $Q_{2 a}$ both transform in the fundamental representation of $\mathrm{SU}(M)_{h}, r \sim M$. If we ignore the constraint on the rank of the representation of the fermions, higher representations lead to higher power of enhancement in axion-photon coupling. Yet high dimensional representations are also more severely constrained by the Planckian Landau pole requirement and pose more challenges to be UV completed in string theory.

- We don't consider models with KSVZ fermions transforming under both $\mathrm{SU}(M)_{h}$ and $\mathrm{SU}(3)_{c}$. Let's take a look at a simple model containing such fermions. Consider a vector-like fermion pair $Q_{1}\left(\widetilde{Q}_{1}\right)$, charged under both $\mathrm{SU}(M)_{h}$ and $\mathrm{SU}(3)_{c}$, coupling to the heavy axion $a_{1}$. To realize the alignment mechanism, we need another vector-like fermion pair $Q_{2}\left(\widetilde{Q}_{2}\right)$, transforming under $\mathrm{SU}(M)_{h}$ and coupling to the second axion $a_{2}$, which is mostly the QCD axion. These fermions will generate axion couplings to the hidden and QCD gluons as

$$
\left[\mu_{h}\left(Q_{1}\right) D_{c}\left(Q_{1}\right) a_{1}+\mu_{h}\left(Q_{2}\right) D_{c}\left(Q_{2}\right) a_{2}\right] H \widetilde{H}+\mu_{c}\left(Q_{1}\right) D_{h}\left(Q_{1}\right) a_{1} G \widetilde{G},
$$

where we only write down the group theoretical factors explicitly. Again we assume these fermions are weak singlets for simplicity. Integrating out the heavy fermions and the heavy axion $a_{1}$, we have the QCD axion-gluon coupling as

$$
\frac{\mu_{c}\left(Q_{1}\right) D_{h}\left(Q_{1}\right) \mu_{h}\left(Q_{2}\right) D_{c}\left(Q_{2}\right)}{\mu_{h}\left(Q_{1}\right) D_{c}\left(Q_{1}\right)} a_{2} G \widetilde{G} \sim \frac{M}{3} \mu_{h}\left(Q_{2}\right) D_{c}\left(Q_{2}\right) a_{2} G \widetilde{G} .
$$

Compared to eq. (2.6), this model does not lead to a parametric enhancement of $f_{a}$ relative to the fundamental period $F_{0}$ in the UV, as we want.

- In general, we do not require the heavy fermions to decay. They could be (meta)stable and phenomenologically viable as long as the inflation scale is below the confinement scale of the hidden gauge group. In the specific model, $Q_{1 a}$ could decay through highdimensional operator $\frac{Q_{1 a} Q_{1 c} Q_{1 c} e^{c} \phi_{1}^{3}}{M_{\mathrm{pl}}^{5}}$ with $e^{c}$ the right-handed lepton in the standard model, which respects the PQ symmetries. Provided $Q_{1 c}$ is lighter than $Q_{1 a}$ and $m_{Q_{1 a}} \gtrsim 10^{15} \mathrm{GeV}$, the lifetime of $Q_{1 a}$ is shorter than $\sim 10^{-2}$ s, so that $Q_{1 a}$ decays before BBN. The other fermions are stable on the cosmological scale. Yet the model could be modified slightly to make the rest of the fermions decay as well. For instance, changing the $\mathrm{U}(1)_{Y}$ assignments of $Q_{1 b}$ and $Q_{2 b}$ to $-1 / 3$ or $2 / 3$ allow dimension-four operators that mix these heavy quarks with the standard model quarks and induce them to decay. These changes won't affect the axion-photon coupling enhancement significantly. 


\begin{tabular}{|c|c|c|c|}
\hline$M$ & $m_{Q}(\mathrm{GeV})$ & $f_{a}(\mathrm{GeV})$ & $r=4 M\left(M^{2}-1\right)$ \\
\hline 3 & $1.3 \times 10^{4}$ & $6.0 \times 10^{5}$ & 96 \\
4 & $5.5 \times 10^{10}$ & $3.3 \times 10^{12}$ & 240 \\
5 & $3.6 \times 10^{13}$ & $2.7 \times 10^{15}$ & 480 \\
6 & $1.0 \times 10^{15}$ & $9.1 \times 10^{16}$ & 840 \\
7 & $7.3 \times 10^{15}$ & $7.5 \times 10^{17}$ & 1344 \\
8 & $2.6 \times 10^{16}$ & $3.0 \times 10^{18}$ & 2016 \\
9 & $5.9 \times 10^{16}$ & $7.7 \times 10^{18}$ & 2880 \\
10 & $1.0 \times 10^{17}$ & $1.5 \times 10^{19}$ & 3960 \\
\hline
\end{tabular}

Table 2. For a given choice of $M$ for an $\mathrm{SU}(M)_{h}$ gauge group, the smallest $m_{Q}$ and corresponding decay constant $f_{a}$ for which we do not hit a Landau pole below $10^{18} \mathrm{GeV}$, and the corresponding enhancement factor $r$ of the axion-photon coupling. We assume all the heavy KSVZ fermions have the same mass $m_{Q}=y F_{0}$ where we have chosen $y=0.2$ as a reference value.

- The domain wall number is 1 in our model because $f_{a}=F_{a}$. Thus the model does not have a potential domain wall problem. In general, the domain wall problem could be solved by introducing a small explicit soft breaking of PQ symmetry that doesn't spoil the axion quality [48].

- Although gauge coupling unification is a nice and desirable feature in general, we will not use it a necessary requirement to restrict the representations and heavy fermion masses in our discussions.

\subsection{Landau pole constraint}

The additional KSVZ vector-like fermions modify the RG running of the SM gauge couplings. In the model in the previous section, all non-Abelian gauge groups are asymptotically free. Yet the charged fermions will accelerate the running of $\mathrm{U}(1)_{Y}$ towards large values and lower its Landau pole. We solve the two-loop RG equations numerically to compute the running of the gauge couplings. The two-loop RG equations could be found in appendix B. For simplicity we will assume that all the $Q$ 's have the same mass and set the hidden gauge coupling to be 1 at the scale of $m_{Q}$. If we demand the Landau poles of $\mathrm{U}(1)_{Y}$ to be above the Planck scale $\left(\gtrsim 10^{18} \mathrm{GeV}\right)$, the minimum allowed vectorlike fermion mass as a function of the degree of the hidden gauge group, $M$, is shown in table 2 . Notice that $m_{Q}=y F_{0}$ which is below the effective decay constant $f_{a}$.

\section{Scenario II: confinement tower}

Now let's extend the bi-axion alignment model to a multi-axion alignment model. We will demonstrate that just as we use KSVZ to UV complete the KNP alignment, we could apply KSVZ to build up a clockwork, which offers a simple way to realize the clockwork structure [33]. 
Consider $n-1$ hidden gauge groups $\left(\mathrm{SU}(M)_{h}\right)^{n-1}$ confining at scales $\Lambda_{1}, \Lambda_{2}, \cdots$, $\Lambda_{n-1} \gg \Lambda_{\mathrm{QCD}}$. There are $n$ PQ fields $\phi_{i}, i=1,2, \cdots, n$, breaking at a common high energy scale $F_{0}>\Lambda$ (we choose a common $F_{0}$ for simplicity), resulting in $n$ axion fields $a_{i}$ 's. The Lagrangian can be schematically written as

$$
\mathcal{L}=\frac{1}{8 \pi F_{0}}\left[\left(\sum_{i=1}^{n-1}\left(M a_{i}+a_{i+1}\right) \alpha_{i} H_{i} \widetilde{H}_{i}\right)+a_{1} \alpha_{s} G \widetilde{G}+a_{n} \alpha_{\mathrm{em}} F \widetilde{F}\right],
$$

where $H_{i}$ is the field strength of the $i$ th $\mathrm{SU}(M)_{h}$. The potential for the axions is

$$
V=\Lambda_{\mathrm{QCD}}^{4} \cos \left(\frac{a_{1}}{F_{0}}\right)+\sum_{i=1}^{n-1} \Lambda_{i}^{4} \cos \left(\frac{M a_{i}+a_{i+1}}{F_{0}}\right) .
$$

Integrating out the heavy axions $a_{1}, a_{2}, \cdots, a_{n-1}$ could be done by setting the arguments $M a_{i}+a_{i+1}=0, i=1, \ldots n-1$. Again in a concrete model, there could be order-one coefficients in front of the axion fields. This leads to

$$
a_{1} \approx \frac{a_{n}}{M^{n-1}},
$$

where we ignore $(-1)^{n-1}$. Then the effective Lagrangian of the lightest axion $a_{n}$, which is identified as the QCD axion, to be

$$
\mathcal{L}=\frac{a_{n}}{8 \pi M^{n-1} F_{0}}\left[\alpha_{s} G \widetilde{G}+M^{n-1} \alpha_{\mathrm{em}} F \widetilde{F}\right]
$$

Thus the effective decay constant is $f_{a}=M^{n-1} F_{0}$. The coefficient of the axion-photon coupling is enhanced by $r=M^{n-1}$, which could be arbitrarily large in principle.

This mechanism could be realized in a KSVZ model easily as well. The particle content of one particular model is shown in table 3. In this model,

$$
r=(2 M)^{n-1} .
$$

For $M=3, n=5$ gives $r=1296$ and $n=9$ gives $r=1.6 \times 10^{6}$. There is no Landau pole issue in this model since only one set of vector-like fermions is charged under $\mathrm{U}(1)_{Y}$. The vector-like fermions and the radial modes of the PQ fields have masses of order $F_{0}$. The heavy axions have masses of order $\Lambda^{2} / F_{0}$. Depending on $M$ and $n$, these particles could be relatively light and may be even close to the TeV scale to be probed directly at the LHC or future colliders. We will leave this model-dependent phenomenology for future work.

The low energy spectrum of axions in our model is very similar to that of the clockwork based on many scalars with a particular type of quartic interaction in ref. [32]. One explicit way to see the similarity is that the mass matrices for the axions in both models take the same tridiagonal form. Yet in our model, the clockwork is based on confining gauge groups and fermions with small representations. This might be more easily realized in the UV than a set of scalars with $1 / 3^{n}$ charge assignments.

The "confinement tower" construction we present here is very similar to a model presented in section III of [33], which differs in representation choice and in being supersymmetric. Our scenario also bears some similarity to models recently proposed in ref. [39], 


\begin{tabular}{|c|cccccc|}
\hline & $\mathrm{SU}(M)_{h ; 1}$ & $\mathrm{SU}(N)_{h ; 2}$ & $\cdots$ & $\mathrm{SU}(N)_{h ; n-1}$ & $\mathrm{SU}(3)_{C}$ & $\mathrm{U}(1)_{Y}$ \\
\hline$\phi_{1}$ & 1 & 1 & 1 & 1 & 1 & 0 \\
$Q_{1 a}\left(\widetilde{Q}_{1 a}\right)$ & $\mathrm{Adj}$ & 1 & 1 & 1 & 1 & 0 \\
$Q_{1 b}\left(\widetilde{Q}_{1 b}\right)$ & 1 & 1 & 1 & 1 & $3(\overline{3})$ & 0 \\
\hline$\phi_{2}$ & 1 & 1 & 1 & 1 & 1 & 0 \\
$Q_{2 a}\left(\widetilde{Q}_{2 a}\right)$ & $M(\bar{M})$ & 1 & 1 & 1 & 1 & 0 \\
$Q_{2 b}\left(\widetilde{Q}_{2 b}\right)$ & 1 & $\mathrm{Adj}$ & 1 & 1 & 1 & 0 \\
\hline$\phi_{3}$ & 1 & 1 & 1 & 1 & 1 & 0 \\
$Q_{3 a}\left(\widetilde{Q}_{3 a}\right)$ & 1 & $M(\bar{M})$ & 1 & 1 & 1 & 0 \\
$Q_{3 b}\left(\widetilde{Q}_{3 b}\right)$ & 1 & 1 & $\mathrm{Adj}$ & 1 & 1 & 0 \\
\hline$\ldots$ & $\cdots$ & $\cdots$ & $\cdots$ & $\cdots$ & $\cdots$ & $\cdots$ \\
\hline$\phi_{n}$ & 1 & 1 & 1 & 1 & 1 & 0 \\
$Q_{n a}\left(\widetilde{Q}_{n a}\right)$ & 1 & 1 & 1 & $M(\bar{M})$ & 1 & 0 \\
$Q_{n b}\left(\widetilde{Q}_{n b}\right)$ & 1 & 1 & 1 & 1 & 1 & $1(-1)$ \\
\hline
\end{tabular}

Table 3. Particle content of a confinement tower model.

in which the axion with a small $F_{0}$ and a large $f_{a}$ arises as a Goldstone from a set of confining gauge groups as well. Our model is KSVZ type with heavy fermions' mass above the confining scale while models in ref. [39] rely on the condensation of fermions. We have several elementary axions while their models involve mostly composite axions.

\subsection{Axion quality}

It is known that a global continuous symmetry is not respected by quantum gravity [49-54] and we generally expect high dimensional operators suppressed by Planck scale that break the global PQ symmetry to appear. These operators tend to generate too large a strong CP phase and ruin the PQ mechanism. One possible way to suppress the dangerous operators, though baroque, is to invoke a discrete symmetry $\mathbb{Z}_{N}$ with $N \gg 1$ to suppress up to dimension 10 operators. While the alignment model based on a single confining gauge group in section 2 is subject to the same issue as the standard KSVZ model, the axion quality in the confinement tower scenario could be significantly improved. This is due to the exponential enhancement of the effective decay constant $f_{a}$ over the fundamental period $F_{0}$ in the UV. For example, consider the dimension-five operator $c_{1} \phi_{n}^{5} / M_{\mathrm{pl}}$ which explicitly breaks the PQ symmetry. It will contribute to the axion potential

$$
\delta V=\frac{\left|c_{1}\right| F_{0}^{5}}{M_{\mathrm{pl}}} \cos \left(\frac{5 a_{n}}{F_{0}}-\alpha\right) \quad \text { with } \quad \alpha=\arg \left[c_{1}\right],
$$

which shifts the strong CP phase by

$$
\delta \theta \approx \alpha \frac{F_{0}}{5 f_{a}} \approx \frac{\alpha}{5 r}
$$


For a generic complex coefficient $c_{1}, \alpha \sim \mathcal{O}(1)$. The larger the enhancement factor $r$ is, the smaller the shift in $\theta$ is. If $r>10^{10}$, the axion is immune to the PQ breaking highdimensional operators. For smaller $r$, the axion quality problem is alleviated such that we only need to introduce some much smaller discrete symmetry to protect the axion. This argument also applies to the original scalar clockwork scenario [42].

\section{Scenario III: kinetic mixing of multiple axions}

A set of axions can, in general, kinetically mix with each other [55]. This idea has found various applications in phenomenology; for instance, it has been used to produce a model in which a $7 \mathrm{keV}$ dark matter axion decays to X-rays by mixing with a lighter QCD axion [56]. Here we will demonstrate that the QCD axion can potentially obtain larger couplings to photons by mixing with a lighter axion field.

To this end, we consider the following model: we have a QCD axion field $a(x)$, a new confining gauge group with field strength $H_{\mu \nu}$, and an axion $b(x)$ that obtains a mass when $H$ confines. We are interested in the limit $m_{a} \gg m_{b}$. We also assume that $b$ couples to photons, and that $a$ and $b$ kinetically mix. The Lagrangian is

$$
\begin{aligned}
\mathcal{L} & =\frac{1}{2} \partial_{\mu} a \partial^{\mu} a+\frac{1}{2} \partial_{\mu} b \partial^{\mu} b+\epsilon \partial_{\mu} a \partial^{\mu} b+c_{b} \frac{\alpha}{8 \pi} \frac{b}{F_{b}} F_{\mu \nu} \widetilde{F}^{\mu \nu}+\frac{\alpha_{H}}{8 \pi} \frac{b}{F_{b}} H_{\mu \nu} \widetilde{H}^{\mu \nu}+\frac{\alpha_{s}}{8 \pi} \frac{a}{F_{a}} G_{\mu \nu} \widetilde{G}^{\mu \nu} \\
& \rightarrow \frac{1}{2} \partial_{\mu} a \partial^{\mu} a+\frac{1}{2} \partial_{\mu} b \partial^{\mu} b+\epsilon \partial_{\mu} a \partial^{\mu} b+c_{b} \frac{\alpha}{8 \pi} \frac{b}{F_{b}} F_{\mu \nu} \widetilde{F}^{\mu \nu}-V_{G}(a)-V_{H}(b) .
\end{aligned}
$$

Because $\epsilon$ is a continuous quantity and $a F \widetilde{F}$ couplings are quantized, it might at first seem that the kinetic mixing cannot induce an effective coupling of the heavy axion $a$ to photons. However, if we diagonalize the kinetic and mass terms we see that the independently propagating axion fields are misaligned with the basis in which the axions have well-defined periodicity, which allows for more general couplings.

The physics is easiest to understand by first imagining the limit in which $b$ remains massless. In this case, following a prescription familiar from the physics of dark photons [57], we eliminate the kinetic mixing with a field redefinition, $b \rightarrow b-\epsilon a$, after which we must divide $a$ by $\sqrt{1-\epsilon^{2}}$ to canonically normalize it. This has the effect of leaving terms like $a G \widetilde{G}$ unchanged, so the heavy field has no admixture of $b$. However, the coupling of $b$ to photons now induces a coupling of the redefined $a$ to photons:

$$
\mathcal{L}_{\text {diag }} \supset\left(\frac{c_{b} \epsilon F_{a}}{F_{b}}+\mathcal{O}\left(\epsilon^{2}\right)\right) \frac{\alpha}{8 \pi} \frac{a}{F_{a}} F_{\mu \nu} \widetilde{F}^{\mu \nu} .
$$

The couplings of the light field $b$ remain quantized after this field redefinition (much as a massless photon always couples to a well-defined conserved current), but the heavier field $a$ acquires a new coupling of order $\epsilon F_{a} / F_{b}$. In particular, if the kinetic mixing is large and if $F_{b} \ll F_{a}$, the mixing contribution to $g_{a \gamma \gamma}$ can overwhelm more direct contributions.

We can now reintroduce the mass of $b$, which will give subleading corrections to the QCD axion's couplings of order $m_{b}^{2} / m_{a}^{2}$ relative to the corrections considered above. It will also allow the lighter field $b$ to obtain non-quantized couplings by mixing with $a$, but these 
will be suppressed not only by $\epsilon$ but also by $m_{b}^{2} / m_{a}^{2}$. For this reason, models where the QCD axion mixes with heavier axions are less effective at modifying its couplings to photons. In that case, keeping $a$ as the QCD axion, we have $m_{b}>m_{a}$ and the photon coupling behaves as $\epsilon\left(F_{a} / F_{b}\right)\left(m_{a}^{2} / m_{b}^{2}\right)$. Hence, it would be difficult to enhance the photon coupling.

\subsection{Realizing large mixing}

In renormalizable KSVZ or DFSZ-like axion models, we expect kinetic mixing effects to generally be small. However, in scenarios where axions come from higher dimensional gauge fields, the topology of internal dimensions can lead to sizable mixing effects. We may have, for instance, a supersymmetric completion containing a variety of dimensionless moduli fields $T_{i}=\tau_{i}+\frac{\mathrm{i}}{2 \pi} \theta_{i}$ where $\theta_{i} \cong \theta_{i}+2 \pi$ are periodic axion fields. The perturbative Kähler potential $K\left(T_{i}+T_{i}^{\dagger}\right)$ depends on the real moduli but not the axions. Axion kinetic terms arise from derivatives of the Kähler potential; if the Kähler potential depends in a sufficiently general way on the $\tau_{i}$, these kinetic terms may be highly mixed. A variety of examples are discussed in [58]. The context is the Type IIB string, where the fields $\tau_{i}$ parametrize the volumes of 4-dimensional cycles within a $6 \mathrm{~d}$ compactification. The Kähler potential depends on the volume of the internal dimensions in string units,

$$
K=-2 M_{\mathrm{Pl}}^{2} \log (\mathcal{V}),
$$

where $\mathcal{V}$ is a function of the $\tau_{i}$. Let us extract a simplified version of one illustrative example from section 4.4 in [58]. We may have

$$
\mathcal{V}=\mathcal{V}_{0}-\alpha_{1} \tau_{1}^{3 / 2}-\beta_{1} \tau_{1}^{1 / 2} \tau_{2}-\beta_{2} \tau_{1} \tau_{2}^{1 / 2}-\alpha_{2} \tau_{2}^{3 / 2}
$$

where $\mathcal{V}_{0}$ is a large overall volume stabilized in a way that effectively decouples from the fields $\tau_{1,2}$, and the $\alpha_{i}$ and $\beta_{i}$ are some order-one coefficients, calculable from topological data (intersection numbers).

In this case, one readily calculates that the kinetic terms for the axions have the form:

$$
\frac{1}{2} F_{1}^{2} \partial_{\mu} \theta_{1} \partial^{\mu} \theta_{1}+\epsilon F_{1} F_{2} \partial_{\mu} \theta_{2} \partial^{\mu} \theta_{2}+\frac{1}{2} F_{2}^{2} \partial_{\mu} \theta_{2} \partial^{\mu} \theta_{2}
$$

with

$$
\begin{aligned}
F_{1}^{2} & \approx \frac{M_{\mathrm{Pl}}^{2}}{\mathcal{V}_{0}} \frac{3 \alpha_{1}\left\langle\tau_{1}\right\rangle-\beta_{1}\left\langle\tau_{2}\right\rangle}{4\left\langle\tau_{1}\right\rangle^{3 / 2}}+\mathcal{O}\left(M_{\mathrm{Pl}}^{2} / \mathcal{V}_{0}^{2}\right), \\
F_{2}^{2} & \approx \frac{M_{\mathrm{Pl}}^{2}}{\mathcal{V}_{0}} \frac{3 \alpha_{2}\left\langle\tau_{2}\right\rangle-\beta_{2}\left\langle\tau_{1}\right\rangle}{4\left\langle\tau_{2}\right\rangle^{3 / 2}}+\mathcal{O}\left(M_{\mathrm{Pl}}^{2} / \mathcal{V}_{0}^{2}\right), \\
\epsilon & \approx \frac{2 \beta_{1}\left\langle\tau_{1}\right\rangle^{1 / 4}\left\langle\tau_{2}\right\rangle^{3 / 4}+2 \beta_{2}\left\langle\tau_{1}\right\rangle^{3 / 4}\left\langle\tau_{2}\right\rangle^{1 / 4}}{\left(3 \alpha_{1}\left\langle\tau_{1}\right\rangle-\beta_{1}\left\langle\tau_{2}\right\rangle\right)^{1 / 2}\left(3 \alpha_{2}\left\langle\tau_{2}\right\rangle-\beta_{2}\left\langle\tau_{1}\right\rangle\right)^{1 / 2}}+\mathcal{O}\left(1 / \mathcal{V}_{0}\right) .
\end{aligned}
$$

In this example we see that:

- If $\mathcal{V}_{0} \gg 1$, all decay constants are well below the Planck scale: the prefactor is set by the string scale $M_{\text {string }} \sim M_{\mathrm{Pl}} / \sqrt{\mathcal{V}_{0}}$. 
- If $\left\langle\tau_{1}\right\rangle \sim\left\langle\tau_{2}\right\rangle$, then the two decay constants are parametrically the same size and their mixing $\epsilon$ is $\mathcal{O}(1)$.

- The example from [58] has $\alpha_{1}, \alpha_{2}, \beta_{1}>0$ and $\beta_{2}<0$. In this case, we can avoid ghosts if $\left\langle\tau_{1}\right\rangle \gg\left\langle\tau_{2}\right\rangle$ but not vice versa. The hierarchy of decay constants in this limit is $F_{2} / F_{1} \sim\left\langle\tau_{1} / \tau_{2}\right\rangle^{3 / 4} \gg 1$ and the kinetic mixing is suppressed by

$$
\epsilon \sim\left\langle\tau_{2} / \tau_{1}\right\rangle^{1 / 4} \sim\left(F_{1} / F_{2}\right)^{1 / 3}
$$

If the axion $\theta_{2}$ obtains a much larger mass than $\theta_{1}$, then the couplings of $\theta_{2}$ to gauge fields to which $\theta_{1}$ couples with order-one strength can be enhanced by the large ratio $\epsilon F_{2} / F_{1} \sim\left(F_{2} / F_{1}\right)^{2 / 3}$.

It is not a stretch to believe that axions can have large kinetic mixing in string theory; the structure of the Kähler potential makes it generic for general enough topology. The ingredient that may be somewhat more tricky to realize is a large hierarchy $\left\langle\tau_{1}\right\rangle \gg\left\langle\tau_{2}\right\rangle$ between the volumes of different cycles. For now, we simply observe that we have transmuted a problem of obtaining large axion couplings into a problem of obtaining geometric hierarchies from moduli stabilization. There is a rich literature on moduli stabilization that makes it plausible that such hierarchies can be realized.

In this discussion we have focused on kinetic mixing between just two axions. In theories with a large number of axions, more dramatic effects may be possible. A recent general analysis of kinetic and Stückelberg mixings for multiple axions, including effects on the field range and couplings, appeared in $[59,60]$. The phenomenon of kinetic alignment can arise, with a randomly chosen kinetic matrix having a very large eigenvalue compared to the typical size of the other eigenvalues [61-64]. This is a distinct phenomenon from KNP alignment, which relies on special structure in the charge assignments of the instantons giving rise to dominant contributions to the axion potential. Kinetic alignment has been studied in the inflationary context, where it provides an interesting test case for arguments for or against the ability of quantum gravity to accommodate super-Planckian field ranges [62, 65-70]. It has not yet been applied to more general axion phenomenology, where new mechanisms for sub-Planckian field ranges are already of interest. We will leave consideration of many-axion kinetic mixing for future work.

\section{$5 \quad$ Results and conclusions}

In figure 1 we show the parameter space of the models in the $m_{a}-g_{a \gamma \gamma}$ plane. The current constraints are shown as gray shaded regions, which arise from evolution of horizontal branch stars [71], from the CAST helioscope [72], and microwave cavities such as ADMX [12, 73-76]. The strongest constraint on light axions arises from non-observation of axions from SN1987A [77] and from conversion of X-ray photons to axions in cluster magnetic fields [78-80]. Observation of black hole spins disfavors a range of axion masses which would lead to superradiance of the black hole [81]. There are additional constraints from observations of the gamma-ray spectra by HESS [82] and Fermi-LAT [83]. 


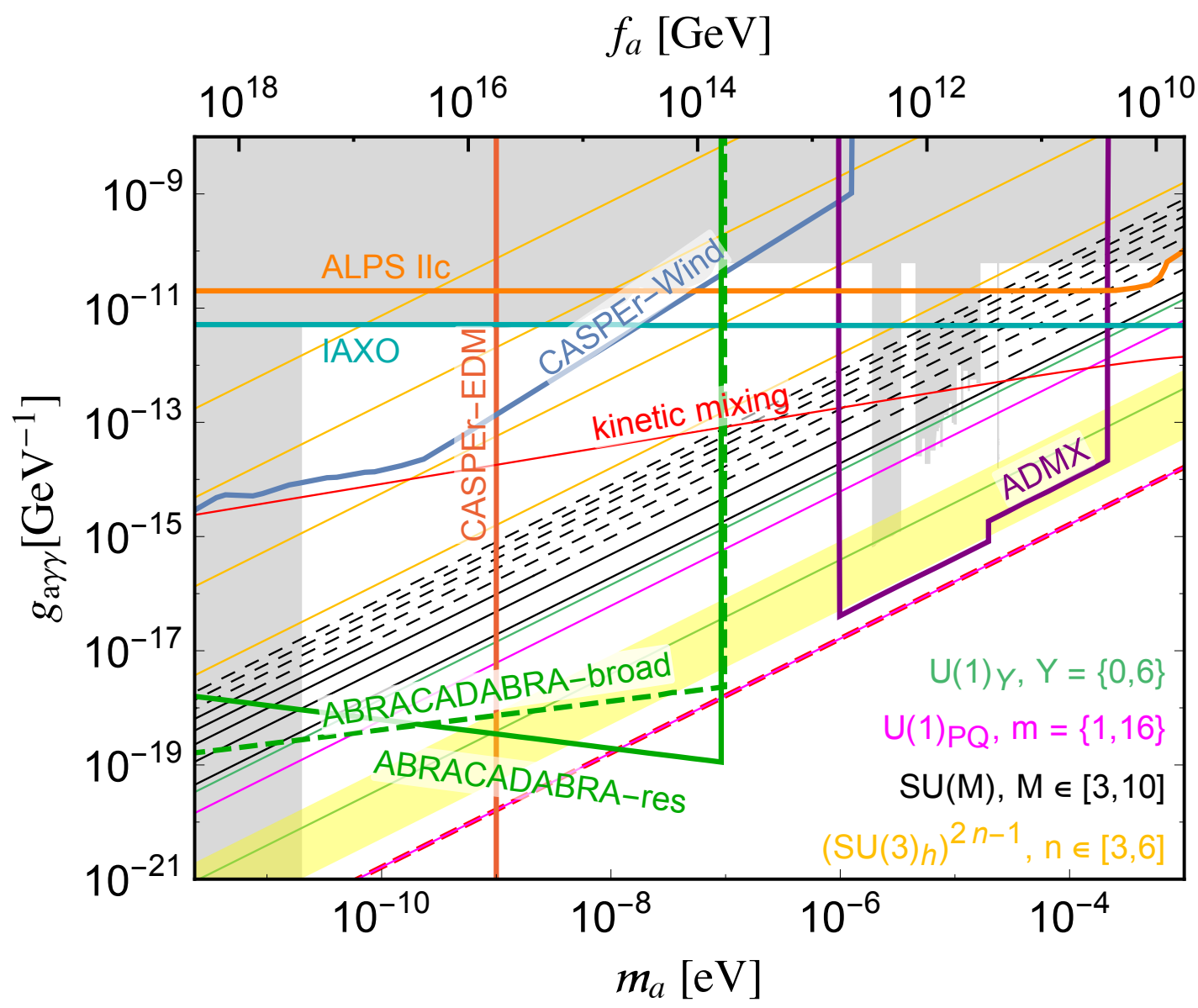

Figure 1. Parameter space of the axion coupling with photons. The gray regions are existing limits. Thin slanted lines represent the predictions in models of sections 2 (black), 3 (amber), and 4 (red) and appendix C (green, magenta) are shown explicitly. Regions bounded by colored lines show sensitivity of upcoming experiments, ALPS II (orange), IAXO (teal), CASPEr-Wind (blue), CASPEr-EDM (red), ABRACADABRA (green) and ADMX (purple). Further details for the plot can be found in the text.

A number of future experiments will cover the unexplored parameter space for the QCD axion and axion-like particles. ADMX will extend its reach to axion dark matter for a wider mass range and to higher sensitivities [84]. The "light shining through walls" experiment ALPS II [85], and the helioscope IAXO [86] will be sensitive to large axion photon couplings. There are new experimental proposals such as CASPEr-EDM and CASPEr-Wind [13, 16, 87] which use NMR, and ABRACADABRA [18] which is a broadband/resonant search for magnetic fluxes induced by axions in a background magnetic field. These experiments can probe light axions down to the QCD line. We show projected sensitivities from future experiments as regions bounded by colored solid lines in figure 1 .

Overlaid on the experimental reach, we show the possible values of $g_{a \gamma \gamma}$ obtained in the models in sections 2, 3 and 4, and in appendix C. The traditional axion-photon coupling band (as discussed in [29]) is shown as the pale yellow shaded region. With black lines we 
show the possible enhancement of $g_{a \gamma \gamma}$ for the model with a single confining gauge group presented in section 2. In this case we have estimated the minimum $f_{a}$ required for the hypercharge Landau pole to be below the Planck scale, and we show $f_{a}$ values smaller than this value as dashed lines. We note however that this is not a strict constraint on the model space, as a lower Landau pole or a different value of the Yukawa coupling is possible. Simple extensions of the KSVZ model with large hypercharges or large PQ charges are also shown, subject to the constraints discussed in appendix C; we see that they can only get moderate enhancements relative to the more traditional KSVZ models. Including the effect of the confining tower clockwork model in section 3 lets us cover the entire parameter space, similar to ref. [32]. We have shown (in amber) a particular realization with varying number of copies of the confining group, which is chosen to be $\mathrm{SU}(3)$. We also show an example of the enhancement we can obtain by kinetically mixing the QCD axion with another lighter axion. For the lighter axion we chose the coupling to photons at the limit with the mass to be $10^{-13} \mathrm{eV}$, i.e. $g_{a \gamma \gamma}=5.34 \times 10^{-12} \mathrm{GeV}^{-1}$. For concreteness we assume that (before the field redefinition to remove mixing) the light axion coupling to the photon is $g_{a \gamma \gamma}=\alpha_{\mathrm{em}} /\left(2 \pi F_{1}\right)$, and the QCD axion coupling is $g_{a \gamma \gamma}=-1.92 \alpha_{\mathrm{em}} /\left(2 \pi F_{2}\right)$, with $F_{2}=f_{a}$. Then, the maximum enhancement as in equation (4.7) is represented by the red line in figure 1 . We note that mixing with a lighter state can lead to significant deviations from the quantized discretuum of $g_{a \gamma \gamma}$. Finally, without tuned contributions from multiple representations or the kinetic mixing, the smallest $g_{a \gamma \gamma}$ that can be obtained simply is expected from $E / N=2$, and is shown as a red dashed line.

The QCD axion remains a very well-motivated dark matter candidate, with exciting upcoming experiments searching for its couplings to photons. We have shown that minimal extensions to the simplest models can lead to a large enhancement of axion-photon couplings, making most of the open parameter space a promising target to look for QCD axions.

\section{Acknowledgments}

MR and LTW thank the 29th Recontres de Blois for providing a congenial environment to begin discussing this topic. A portion of this work was carried out at the Aspen Center for Physics, which is supported by the National Science Foundation grant PHY-1607611. PA is supported by the NSF grants PHY-0855591 and PHY-1216270. JF is supported by the DOE grant DE-SC-0010010. MR is supported in part by the DOE grant DE-SC0013607 and the NASA grant NNX16AI12G. LTW is supported by the DOE grant DE-SC0013642.

\section{A Mass mixing and non-quantized couplings}

In the introduction we asserted that couplings of the form $a F \widetilde{F}$ are quantized, but then displayed an axion-pion mixing effect (1.4) that appeared to violate the quantization. For clarity, we will explain this apparent contradiction. The short answer is that such couplings are part of a larger set of couplings summing up to a periodic function of the axion, and as such are always accompanied by a factor of $m_{a}^{2}$ as a spurion for the breaking of the 
continuous shift symmetry. In particular, it is only because $\Lambda_{\mathrm{QCD}}^{4} \sim m_{a}^{2} F_{a}^{2}$ that this effect is sizable. More general mass mixing will, as a rule, lead to negligible non-quantized effects.

Consider the following very schematic toy model for axion-meson mixing, which simplifies the situation in real QCD by considering only a single meson, which we denote $\pi^{0}$ (though in the one flavor case it behaves more like the $\eta^{\prime}$; the full theory includes several mesons that all mix). We assume this meson couples to photons through a Lagrangian

$$
\mathcal{L}_{\text {int }}=\frac{\alpha}{8 \pi} \frac{\pi^{0}}{f} F_{\mu \nu} \widetilde{F}^{\mu \nu}+\Lambda^{4} \cos \left(\frac{\pi^{0}}{f}+\frac{a}{F_{a}}\right)+m_{q} \mu^{3} \cos \left(\frac{\pi^{0}}{f}\right),
$$

which has the desired property that when $m_{q} \rightarrow 0$ there is a massless axion field. Here $\Lambda$ is roughly the confinement scale, and $\mu^{3}$ is $|\langle q \bar{q}\rangle|$. Our goal is to see, after integrating out the $\pi^{0}$, what form the axion coupling to photons has in the low-energy effective theory. This serves to illustrate the important physics for the QCD mixing contribution to the axion-photon coupling, without all of the details.

First, notice that in the $m_{q} \rightarrow 0$ limit, we can integrate out the $\pi^{0}$ and find a coupling $-\frac{\alpha}{8 \pi} \frac{a}{F_{a}} F \widetilde{F}$, which has an integer coefficient as expected from (1.1). The physics at $m_{q} \neq 0$ is more interesting: if we expand the potential to quadratic order and minimize, we find

$$
\frac{\pi^{0}}{f}=-\frac{\Lambda^{4}}{\Lambda^{4}+m_{q} \mu^{3}} \frac{a}{F_{a}} \approx-\frac{a}{F_{a}}+\frac{m_{q} \mu^{3}}{\Lambda^{4}} \frac{a}{F_{a}}+\cdots,
$$

where in the last step we see that if we expand at small quark mass we obtain an apparently small shift away from integer values of the coefficient of $\frac{\alpha}{8 \pi} \frac{a}{F_{a}} F \widetilde{F}$ in the effective theory. How is this consistent with the period of the field $a$ ? The answer comes from keeping the full set of nonlinear interactions: the condition $\partial V / \partial \pi^{0}=0$ requires

$$
\frac{\pi^{0}}{f}=-\arctan \frac{\Lambda^{4} \sin \frac{a}{F_{a}}}{\Lambda^{4} \cos \frac{a}{F_{a}}+m_{q} \mu^{3}},
$$

so when we integrate out the $\pi^{0}$ exactly we obtain a coupling of the form $\frac{\alpha}{8 \pi} g\left(a / F_{a}\right) F \widetilde{F}$ where $g(x)$ is a function with period $2 \pi$ and is perfectly consistent with the periodicity of the axion.

This shows that if we consider an effective theory with general couplings

$$
\mathcal{L} \supset\left(c_{1} \frac{a}{F_{a}}+c_{2} \frac{a^{2}}{F_{a}^{2}}+c_{3} \frac{a^{3}}{F_{a}^{3}}+\ldots\right) F_{\mu \nu} \widetilde{F}^{\mu \nu},
$$

there is in general no consistency condition on individual couplings $c_{i}$; rather, they can correspond to the Taylor series of any periodic function, and there is no need to impose $c_{1}=n \alpha /(8 \pi)$ for integer $n$. However, there is a catch: these nonperiodic effects are always proportional to the axion mass squared. The reason is that they are sensitive to the periodicity of the axion, which means they feel the breaking of the continuous axion shift symmetry to a discrete shift symmetry. Such effects always arise from instantons, which in general contribute to the axion mass. In the current context, this is manifested in the proportionality of the non-quantized coefficient in (A.3) to $m_{q} \mu^{3} \sim m_{a}^{2} F_{a}^{2}$. This 
can be a significant effect in QCD because the same source of nonperturbative dynamics gives mass to both the pion and the axion. Other new physics at a scale $\Lambda \approx \Lambda_{\mathrm{QCD}}$ could potentially also affect the axion couplings significantly, but would tend to spoil the strong CP solution. As a result, we do not expect mass mixing to generate significant non-quantized axion-photon couplings.

\section{B Two-loop RG equations for the model in section 2}

The two-loop RG equations for the gauge couplings, $g_{i}$, are given by

$$
\frac{d \alpha_{i}^{-1}}{d t}=-a_{i}-\sum_{j} \frac{b_{i j}}{4 \pi} \alpha_{j}, \quad i=1,2,3, h
$$

where $\alpha_{i}=\frac{g_{i}^{2}}{4 \pi}$ and $t=\frac{1}{2 \pi} \log \frac{\mu}{m_{Z}}$. The one- and two-loop beta functions are [88]

$$
\begin{aligned}
a_{i}= & -\frac{11}{3} C_{2}\left(G_{i}\right)+\frac{4}{3} \sum_{F} \kappa \mu\left(F_{i}\right)+\frac{1}{3} \sum_{S} \eta \mu\left(S_{i}\right) \\
b_{i j}= & -\left(\frac{34}{3} C_{2}\left(G_{i}\right)^{2}-\frac{20}{3} \sum_{F} \kappa C_{2}\left(G_{i}\right) \mu\left(F_{i}\right)-\frac{2}{3} \sum_{S} \eta C_{2}\left(G_{i}\right) \mu\left(S_{i}\right)\right) \delta_{i j} \\
& +4\left(\sum_{F} \kappa C_{2}\left(F_{j}\right) \mu\left(F_{i}\right)+\sum_{S} \kappa C_{2}\left(S_{j}\right) \mu\left(S_{i}\right)\right)
\end{aligned}
$$

where $F$ 's are fermions and $S$ 's are scalars. There is no summation over index $i . \kappa=1(1 / 2)$ for Dirac (Weyl) fermions and $\eta=1(1 / 2)$ for complex (real) scalars. $G_{i}$ denotes the $i$ th gauge factor. $C_{2}$ 's are the Casimir of a given irreducible representation. The Dynkin indices $\mu$ 's include multiplicity factors.

Below $m_{Q}$ but above the confinement scale of $H$, the SM particles are decoupled from the $\mathrm{SU}(M)$ gluons and their contributions to the beta functions are

$$
a=\left(\frac{41}{10},-\frac{19}{6},-7,-\frac{11}{3} M\right)
$$

and

$$
b=\left[\begin{array}{cccc}
\frac{199}{50} & \frac{27}{10} & \frac{44}{5} & 0 \\
\frac{9}{10} & \frac{35}{6} & 12 & 0 \\
\frac{11}{10} & \frac{9}{2} & -26 & 0 \\
0 & 0 & 0 & -\frac{34}{3} M^{2}
\end{array}\right] .
$$

Notice that we use the GUT normalization of the hypercharge coupling, $g_{1}$.

Above $m_{Q}$, the contributions of the heavy vector-like fermions in section 2.1 to the beta functions are

$$
\delta a=\left(\frac{4}{5}\left(M^{2}-1\right), 0, \frac{4}{3}, \frac{8}{3} M+\frac{2}{3}\right)
$$


and

$$
\delta b=\left[\begin{array}{cccc}
\frac{36}{25}\left(M^{2}-1\right) & 0 & 0 & \frac{12}{5} M\left(M^{2}-1\right) \\
0 & 0 & 0 & 0 \\
0 & 0 & \frac{76}{3} & 0 \\
\frac{12}{5} M & 0 & 0 & \frac{64}{3} M^{2}+\frac{13}{3} M-\frac{1}{M}
\end{array}\right] .
$$

We set the gauge coupling of $\mathrm{SU}(M)_{h}$ to be 1 at $m_{Q}$. We ignore the contributions of the Yukawa couplings to the running of the gauge couplings. The Yukawa couplings lead to a much more complicated formula, which we don't include here. The Yukawa couplings between the heavy fermions and the PQ fields are free parameters and could be small. They only contribute to the gauge coupling running at the two-loop order and the effect is numerically tested to be small as long as the they are $\lesssim 1$. (In particular, we have included the Standard Model top Yukawa in the RGEs, and found no change in our conclusions about Landau poles.)

\section{Vector-like leptons with large PQ charge}

In this appendix, we consider the possibility of a large PQ-charged state enhancing the axion-photon coupling. It is similar to the large hypercharge case in using some large charge to increase the coupling yet suffers from different phenomenological issues, which we discuss in some detail below. We study a variant of the KSVZ model with vector-like fermions which carry the large PQ charge. Consider the following matter charge assignment with a global $\mathrm{U}(1)_{\mathrm{PQ}}$,

\begin{tabular}{|c|ccc|}
\hline & $\mathrm{SU}(3)_{c}$ & $\mathrm{U}(1)_{Y}$ & $\mathrm{U}(1)_{\mathrm{PQ}}$ \\
\hline$Q$ & 3 & 0 & 1 \\
$\widetilde{Q}$ & $\overline{3}$ & 0 & 1 \\
$L$ & 1 & 1 & $m$ \\
$\widetilde{L}$ & 1 & -1 & $m$ \\
$\phi$ & 1 & 0 & -2 \\
\hline
\end{tabular}

where $m$ is a positive integer. All the fermions are taken to be weak singlets.

The Lagrangian consistent with these symmetries is

$$
\mathcal{L}=-V(\phi)+\left(\lambda \phi Q \widetilde{Q}+\lambda^{\prime} \frac{\phi^{m}}{\Lambda^{m-1}} L \widetilde{L}+\text { h.c. }\right) .
$$

As usual, $V(\phi)$ is chosen to give the PQ scalar a VEV. The Goldstone can be parametrized as

$$
\phi=F_{a} \mathrm{e}^{\mathrm{i} \frac{a}{F_{a}}}
$$

and the axion excitation around this VEV can be written as,

$$
\lambda \mathrm{e}^{\mathrm{i} \frac{a}{F_{a}}} Q \widetilde{Q}+\lambda^{\prime} \frac{F_{a}^{m}}{\Lambda^{m-1}} \mathrm{e}^{\mathrm{i} \frac{m a}{F_{a}}} L \widetilde{L}+V(\phi) .
$$


In this model, the effective decay constant $f_{a}$ is equal to the fundamental period $F_{a}$. Below we will only use $f_{a}$. Upon doing the chiral rotations to get rid of the phase in the mass terms, and integrating out the heavy fields, we get,

$$
\frac{\alpha_{s}}{8 \pi f_{a}} a G_{\mu \nu}^{a} \widetilde{G}^{a, \mu \nu}+\frac{m \alpha_{\mathrm{em}}}{4 \pi f_{a}} a F_{\mu \nu} \widetilde{F}^{\mu \nu} .
$$

The potential problem here is that the mass for $L, \widetilde{L}$ is suppressed by $\left(f_{a} / \Lambda\right)^{m-1}$. We consider two examples of UV-completing the higher dimensional operator to see how large $m$ can be.

\section{C.1 A chain of vector-like fermions}

A simple renormalizable UV completion of this model is to consider a chain of interactions,

$$
y \phi L \widetilde{N}_{1}+y \phi N_{m-1} \widetilde{L}+\sum_{i=2}^{m-2} y \phi N_{i} \widetilde{N}_{i+1}+\sum_{i=1}^{m-1} M N_{i} \widetilde{N}_{i},
$$

where the charge assignment for $N_{i}, \widetilde{N}_{i}$ is

\begin{tabular}{|c|ccc|}
\hline & $\mathrm{SU}(3)_{c}$ & $\mathrm{U}(1)_{Y}$ & $\mathrm{U}(1)_{\mathrm{PQ}}$ \\
\hline$N_{i}$ & 1 & 1 & $m-2 i$ \\
$\widetilde{N}_{i}$ & 1 & -1 & $-(m-2 i)$ \\
\hline
\end{tabular}

For simplicity, we have kept the mass and the Yukawa couplings of $N_{i}$ the same. Integrating out the $N_{i}$ at the scale $M$, we see that we can identify $\Lambda=M / y$, and $\lambda^{\prime}=y$. If $M$ is smaller or close to $f$ then the solution is essentially the same as adding a particle with a large hypercharge. The advantage of choosing $M>f$ is that the hypercharge Landau pole is postponed.

\section{C.2 Clockwork}

Another possibility is a UV completion similar to the clockwork mechanism. We have $l$ scalar fields $\phi_{i}$ with the interaction terms

$$
\mathcal{L}=\lambda^{\prime} \phi_{0} L \widetilde{L}+\lambda \phi_{l} Q \widetilde{Q}+\sum_{i=0}^{l-1} \kappa \phi_{i}^{\dagger} \phi_{i+1}^{q}+M^{2} \phi_{i}^{\dagger} \phi_{i} .
$$

The state $\phi_{l}$ has PQ charge 2 , as evidenced by its coupling to $Q \widetilde{Q}$. The scalar $\phi_{0}$ has a PQ charge of $2 q^{l} \equiv 2 m$. The mass eigenstate that is light compared to $M$ is identified as $\phi=\phi_{l}$. Thus, integrating out the other $\phi_{i}$ at scale $M$,

$$
\mathcal{L}=\lambda^{\prime}\left(\frac{\kappa \phi}{M^{2}}\right)^{m} L \widetilde{L}+\lambda \phi Q \widetilde{Q}
$$

The effective hierarchy we get in this case is $q^{l}$, at the cost of introducing only $l$ new fields with hypercharge. This model reduces to the single axion model above only if $M>f_{a}$, 
such that all but one axion can be integrated out. For $M \ll f_{a}$ the model has multiple axions clockworking.

In both the mechanisms above, we see that if we want to integrate out heavier physics to get a single axion effective theory at the scale $f_{a}$, the mass of the $L, \widetilde{L}$ fermion is suppressed exponentially. If we impose the condition that the fermions $L, \widetilde{L}$ are heavier than about $1 \mathrm{TeV}$,

$$
\frac{f_{a}}{M} \geq\left(\frac{1 \mathrm{TeV}}{M}\right)^{\frac{1}{m}} \geq\left(\frac{1 \mathrm{TeV}}{M_{\mathrm{pl}}}\right)^{\frac{1}{m}} \simeq 10^{-\frac{16}{m}}
$$

where we have chosen $y \simeq 1, \kappa \simeq M$ for simplicity. Therefore, we see that we can only get the enhancement factor $r=2 m<32$ if we want some hierarchy between the scales $f_{a}$ and $M$.

Open Access. This article is distributed under the terms of the Creative Commons Attribution License (CC-BY 4.0), which permits any use, distribution and reproduction in any medium, provided the original author(s) and source are credited.

\section{References}

[1] R.D. Peccei and H.R. Quinn, Constraints Imposed by CP Conservation in the Presence of Instantons, Phys. Rev. D 16 (1977) 1791 [INSPIRE].

[2] R.D. Peccei and H.R. Quinn, CP Conservation in the Presence of Instantons, Phys. Rev. Lett. 38 (1977) 1440 [INSPIRE].

[3] F. Wilczek, Problem of Strong $p$ and $t$ Invariance in the Presence of Instantons, Phys. Rev. Lett. 40 (1978) 279 [INSPIRE].

[4] S. Weinberg, A New Light Boson?, Phys. Rev. Lett. 40 (1978) 223 [InSPIRE].

[5] J.E. Kim, Weak Interaction Singlet and Strong CP Invariance, Phys. Rev. Lett. 43 (1979) 103 [INSPIRE].

[6] M.A. Shifman, A.I. Vainshtein and V.I. Zakharov, Can Confinement Ensure Natural CP Invariance of Strong Interactions?, Nucl. Phys. B 166 (1980) 493 [INSPIRE].

[7] A.R. Zhitnitsky, On Possible Suppression of the Axion Hadron Interactions (in Russian), Sov. J. Nucl. Phys. 31 (1980) 260 [inSPIRE].

[8] M. Dine, W. Fischler and M. Srednicki, A Simple Solution to the Strong CP Problem with a Harmless Axion, Phys. Lett. B 104 (1981) 199 [INSPIRE].

[9] J. Preskill, M.B. Wise and F. Wilczek, Cosmology of the Invisible Axion, Phys. Lett. B 120 (1983) 127 [INSPIRE].

[10] M. Dine and W. Fischler, The Not So Harmless Axion, Phys. Lett. B 120 (1983) 137 [INSPIRE].

[11] L.F. Abbott and P. Sikivie, A Cosmological Bound on the Invisible Axion, Phys. Lett. B 120 (1983) 133 [INSPIRE].

[12] ADMX collaboration, S.J. Asztalos et al., A SQUID-based microwave cavity search for dark-matter axions, Phys. Rev. Lett. 104 (2010) 041301 [arXiv:0910.5914] [INSPIRE].

[13] P.W. Graham and S. Rajendran, New Observables for Direct Detection of Axion Dark Matter, Phys. Rev. D 88 (2013) 035023 [arXiv:1306.6088] [INSPIRE]. 
[14] E. Armengaud et al., Conceptual Design of the International Axion Observatory (IAXO), 2014 JINST 9 T05002 [arXiv:1401.3233] [INSPIRE].

[15] D. Horns, J. Jaeckel, A. Lindner, A. Lobanov, J. Redondo and A. Ringwald, Searching for WISPy Cold Dark Matter with a Dish Antenna, JCAP 04 (2013) 016 [arXiv:1212.2970] [INSPIRE].

[16] D. Budker, P.W. Graham, M. Ledbetter, S. Rajendran and A. Sushkov, Proposal for a Cosmic Axion Spin Precession Experiment (CASPEr), Phys. Rev. X 4 (2014) 021030 [arXiv: 1306.6089] [INSPIRE].

[17] A. Arvanitaki and A.A. Geraci, Resonantly Detecting Axion-Mediated Forces with Nuclear Magnetic Resonance, Phys. Rev. Lett. 113 (2014) 161801 [arXiv:1403.1290] [INSPIRE].

[18] Y. Kahn, B.R. Safdi and J. Thaler, Broadband and Resonant Approaches to Axion Dark Matter Detection, Phys. Rev. Lett. 117 (2016) 141801 [arXiv:1602.01086] [InSPIRE].

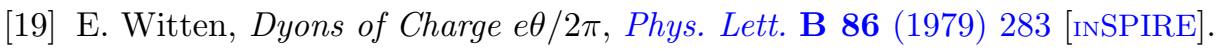

[20] E. Witten, Large-N Chiral Dynamics, Annals Phys. 128 (1980) 363 [INSPIRE].

[21] E. Witten, Theta dependence in the large- $N$ limit of four-dimensional gauge theories, Phys. Rev. Lett. 81 (1998) 2862 [hep-th/9807109] [INSPIRE].

[22] D. Tong, Line Operators in the Standard Model, JHEP 07 (2017) 104 [arXiv:1705.01853] [INSPIRE].

[23] D.B. Kaplan, Opening the Axion Window, Nucl. Phys. B 260 (1985) 215 [INSPIRE].

[24] M. Srednicki, Axion Couplings to Matter. 1. CP Conserving Parts, Nucl. Phys. B 260 (1985) 689 [INSPIRE].

[25] H. Georgi, D.B. Kaplan and L. Randall, Manifesting the Invisible Axion at Low-energies, Phys. Lett. B 169 (1986) 73 [inSPIRE].

[26] P. Svrček and E. Witten, Axions In String Theory, JHEP 06 (2006) 051 [hep-th/0605206] [INSPIRE].

[27] G. Grilli di Cortona, E. Hardy, J. Pardo Vega and G. Villadoro, The QCD axion, precisely, JHEP 01 (2016) 034 [arXiv: 1511.02867] [inSPIRE].

[28] G.F. Giudice, R. Rattazzi and A. Strumia, Unificaxion, Phys. Lett. B 715 (2012) 142 [arXiv: 1204.5465] [INSPIRE].

[29] J.E. Kim, Constraints on very light axions from cavity experiments, Phys. Rev. D 58 (1998) 055006 [hep-ph/9802220] [INSPIRE].

[30] L. Di Luzio, F. Mescia and E. Nardi, Redefining the Axion Window, Phys. Rev. Lett. 118 (2017) 031801 [arXiv:1610.07593] [INSPIRE].

[31] L. Di Luzio, F. Mescia and E. Nardi, Window for preferred axion models, Phys. Rev. D 96 (2017) 075003 [arXiv: 1705.05370] [INSPIRE].

[32] M. Farina, D. Pappadopulo, F. Rompineve and A. Tesi, The photo-philic QCD axion, JHEP 01 (2017) 095 [arXiv:1611.09855] [INSPIRE].

[33] K. Choi and S.H. Im, Realizing the relaxion from multiple axions and its UV completion with high scale supersymmetry, JHEP 01 (2016) 149 [arXiv:1511.00132] [INSPIRE].

[34] D.E. Kaplan and R. Rattazzi, Large field excursions and approximate discrete symmetries from a clockwork axion, Phys. Rev. D 93 (2016) 085007 [arXiv: 1511.01827] [INSPIRE]. 
[35] G. Dvali, Black Holes and Large-N Species Solution to the Hierarchy Problem, Fortsch. Phys. 58 (2010) 528 [arXiv:0706.2050] [inSPIRE].

[36] K. Choi, H. Kim and S. Yun, Natural inflation with multiple sub-Planckian axions, Phys. Rev. D 90 (2014) 023545 [arXiv: 1404.6209] [INSPIRE].

[37] J.E. Kim, H.P. Nilles and M. Peloso, Completing natural inflation, JCAP 01 (2005) 005 [hep-ph/0409138] [INSPIRE].

[38] P. Agrawal, G. Marques-Tavares and W. Xue, Opening up the QCD axion window, arXiv: 1708.05008 [INSPIRE].

[39] R. Coy, M. Frigerio and M. Ibe, Dynamical Clockwork Axions, JHEP 10 (2017) 002 [arXiv: 1706. 04529] [INSPIRE].

[40] L.E. Ibanez and A.M. Uranga, String theory and particle physics: An introduction to string phenomenology, Cambridge University Press (2012) [INSPIRE].

[41] T. Higaki, K.S. Jeong, N. Kitajima and F. Takahashi, The QCD Axion from Aligned Axions and Diphoton Excess, Phys. Lett. B 755 (2016) 13 [arXiv:1512.05295] [INSPIRE].

[42] T. Higaki, K.S. Jeong, N. Kitajima and F. Takahashi, Quality of the Peccei-Quinn symmetry in the Aligned QCD Axion and Cosmological Implications, JHEP 06 (2016) 150 [arXiv: 1603.02090] [INSPIRE].

[43] S.H.H. Tye and S.S.C. Wong, Helical Inflation and Cosmic Strings, arXiv:1404.6988 [INSPIRE].

[44] R. Kappl, S. Krippendorf and H.P. Nilles, Aligned Natural Inflation: Monodromies of two Axions, Phys. Lett. B 737 (2014) 124 [arXiv:1404.7127] [INSPIRE].

[45] I. Ben-Dayan, F.G. Pedro and A. Westphal, Hierarchical Axion Inflation, Phys. Rev. Lett. 113 (2014) 261301 [arXiv:1404.7773] [INSPIRE].

[46] Y. Bai and B.A. Stefanek, Natural millicharged inflation, Phys. Rev. D 91 (2015) 096012 [arXiv: 1405.6720] [INSPIRE].

[47] A. de la Fuente, P. Saraswat and R. Sundrum, Natural Inflation and Quantum Gravity, Phys. Rev. Lett. 114 (2015) 151303 [arXiv:1412.3457] [INSPIRE].

[48] P. Sikivie, Of Axions, Domain Walls and the Early Universe, Phys. Rev. Lett. 48 (1982) 1156 [INSPIRE].

[49] L.F. Abbott and M.B. Wise, Wormholes and Global Symmetries, Nucl. Phys. B 325 (1989) 687 [INSPIRE].

[50] S.R. Coleman and K.-M. Lee, Wormholes made without massless matter fields, Nucl. Phys. B 329 (1990) 387 [INSPIRE].

[51] M. Kamionkowski and J. March-Russell, Planck scale physics and the Peccei-Quinn mechanism, Phys. Lett. B 282 (1992) 137 [hep-th/9202003] [INSPIRE].

[52] R. Kallosh, A.D. Linde, D.A. Linde and L. Susskind, Gravity and global symmetries, Phys. Rev. D 52 (1995) 912 [hep-th/9502069] [INSPIRE].

[53] T. Banks and N. Seiberg, Symmetries and Strings in Field Theory and Gravity, Phys. Rev. D 83 (2011) 084019 [arXiv: 1011.5120] [INSPIRE].

[54] R. Alonso and A. Urbano, Wormholes and masses for Goldstone bosons, arXiv:1706.07415 [INSPIRE]. 
[55] K.S. Babu, S.M. Barr and D. Seckel, Axion dissipation through the mixing of Goldstone bosons, Phys. Lett. B 336 (1994) 213 [hep-ph/9406308] [INSPIRE].

[56] T. Higaki, N. Kitajima and F. Takahashi, Hidden axion dark matter decaying through mixing with QCD axion and the $3.5 \mathrm{keV} X$-ray line, JCAP 12 (2014) 004 [arXiv:1408.3936] [INSPIRE].

[57] B. Holdom, Two U(1)'s and Epsilon Charge Shifts, Phys. Lett. B 166 (1986) 196 [inSPIRE].

[58] M. Cicoli, M. Goodsell and A. Ringwald, The type IIB string axiverse and its low-energy phenomenology, JHEP 10 (2012) 146 [arXiv:1206.0819] [INSPIRE].

[59] G. Shiu, W. Staessens and F. Ye, Widening the Axion Window via Kinetic and Stückelberg Mixings, Phys. Rev. Lett. 115 (2015) 181601 [arXiv:1503.01015] [inSPIRE].

[60] G. Shiu, W. Staessens and F. Ye, Large Field Inflation from Axion Mixing, JHEP 06 (2015) 026 [arXiv: 1503. 02965] [INSPIRE].

[61] T.C. Bachlechner, M. Dias, J. Frazer and L. McAllister, Chaotic inflation with kinetic alignment of axion fields, Phys. Rev. D 91 (2015) 023520 [arXiv:1404.7496] [INSPIRE].

[62] T.C. Bachlechner, C. Long and L. McAllister, Planckian Axions in String Theory, JHEP 12 (2015) 042 [arXiv: 1412.1093] [INSPIRE].

[63] T.C. Bachlechner, K. Eckerle, O. Janssen and M. Kleban, Axions of Evil, arXiv:1703.00453 [INSPIRE].

[64] T.C. Bachlechner, K. Eckerle, O. Janssen and M. Kleban, Systematics of Aligned Axions, JHEP 11 (2017) 036 [arXiv:1709.01080] [INSPIRE].

[65] T. Rudelius, On the Possibility of Large Axion Moduli Spaces, JCAP 04 (2015) 049 [arXiv:1409.5793] [INSPIRE].

[66] T.C. Bachlechner, C. Long and L. McAllister, Planckian Axions and the Weak Gravity Conjecture, JHEP 01 (2016) 091 [arXiv: 1503.07853] [INSPIRE].

[67] M. Montero, A.M. Uranga and I. Valenzuela, Transplanckian axions!?, JHEP 08 (2015) 032 [arXiv: 1503.03886] [INSPIRE].

[68] J. Brown, W. Cottrell, G. Shiu and P. Soler, On Axionic Field Ranges, Loopholes and the Weak Gravity Conjecture, JHEP 04 (2016) 017 [arXiv: 1504.00659] [INSPIRE].

[69] D. Junghans, Large-Field Inflation with Multiple Axions and the Weak Gravity Conjecture, JHEP 02 (2016) 128 [arXiv: 1504.03566] [INSPIRE].

[70] B. Heidenreich, M. Reece and T. Rudelius, Weak Gravity Strongly Constrains Large-Field Axion Inflation, JHEP 12 (2015) 108 [arXiv:1506.03447] [INSPIRE].

[71] D. Cadamuro and J. Redondo, Cosmological bounds on pseudo Nambu-Goldstone bosons, JCAP 02 (2012) 032 [arXiv:1110.2895] [INSPIRE].

[72] CAST collaboration, V. Anastassopoulos et al., New CAST Limit on the Axion-Photon Interaction, Nature Phys. 13 (2017) 584 [arXiv:1705.02290] [INSPIRE].

[73] S. De Panfilis et al., Limits on the Abundance and Coupling of Cosmic Axions at $4.5<m_{a}<5.0 \mu \mathrm{eV}$, Phys. Rev. Lett. 59 (1987) 839 [INSPIRE].

[74] W. Wuensch et al., Results of a Laboratory Search for Cosmic Axions and Other Weakly Coupled Light Particles, Phys. Rev. D 40 (1989) 3153 [INSPIRE]. 
[75] C. Hagmann, P. Sikivie, N.S. Sullivan and D.B. Tanner, Results from a search for cosmic axions, Phys. Rev. D 42 (1990) 1297 [InSPIRE].

[76] B.M. Brubaker et al., First results from a microwave cavity axion search at $24 \mu \mathrm{eV}$, Phys. Rev. Lett. 118 (2017) 061302 [arXiv:1610.02580] [INSPIRE].

[77] A. Payez, C. Evoli, T. Fischer, M. Giannotti, A. Mirizzi and A. Ringwald, Revisiting the SN1987A gamma-ray limit on ultralight axion-like particles, JCAP 02 (2015) 006 [arXiv:1410.3747] [INSPIRE].

[78] M. Berg et al., Constraints on Axion-Like Particles from X-ray Observations of NGC1275, Astrophys. J. 847 (2017) 101 [arXiv: 1605. 01043] [INSPIRE].

[79] M.C.D. Marsh, H.R. Russell, A.C. Fabian, B.P. McNamara, P. Nulsen and C.S. Reynolds, A New Bound on Axion-Like Particles, JCAP 12 (2017) 036 [arXiv:1703.07354] [INSPIRE].

[80] J.P. Conlon, F. Day, N. Jennings, S. Krippendorf and M. Rummel, Constraints on Axion-Like Particles from Non-Observation of Spectral Modulations for X-ray Point Sources, JCAP 07 (2017) 005 [arXiv: 1704.05256] [INSPIRE].

[81] A. Arvanitaki, M. Baryakhtar and X. Huang, Discovering the QCD Axion with Black Holes and Gravitational Waves, Phys. Rev. D 91 (2015) 084011 [arXiv:1411.2263] [InSPIRE].

[82] H.E.S.S. collaboration, A. Abramowski et al., Constraints on axionlike particles with H.E.S.S. from the irregularity of the PKS 2155-304 energy spectrum, Phys. Rev. D 88 (2013) 102003 [arXiv: 1311.3148] [INSPIRE].

[83] Fermi-LAT collaboration, M. Ajello et al., Search for Spectral Irregularities due to Photon-Axionlike-Particle Oscillations with the Fermi Large Area Telescope, Phys. Rev. Lett. 116 (2016) 161101 [arXiv:1603.06978] [INSPIRE].

[84] T.M. Shokair et al., Future Directions in the Microwave Cavity Search for Dark Matter Axions, Int. J. Mod. Phys. A 29 (2014) 1443004 [arXiv:1405.3685] [INSPIRE].

[85] R. Bähre et al., Any light particle search II - Technical Design Report, 2013 JINST 8 T09001 [arXiv: 1302 .5647] [INSPIRE].

[86] J.K. Vogel et al., IAXO - The International Axion Observatory, in 8th Patras Workshop on Axions, WIMPs and WISPs (AXION-WIMP 2012), Chicago, Illinois, 18-22 July 2012 [arXiv: 1302 .3273] [http://1ss.fnal.gov/archive/2013/pub/fermilab-pub-13-699-a.pdf].

[87] A. Garcon et al., The Cosmic Axion Spin Precession Experiment (CASPEr): a dark-matter search with nuclear magnetic resonance, arXiv:1707.05312 [INSPIRE].

[88] M.E. Machacek and M.T. Vaughn, Two Loop Renormalization Group Equations in a General Quantum Field Theory. 1. Wave Function Renormalization, Nucl. Phys. B 222 (1983) 83 [INSPIRE]. 